\title{
The impact of COVID-19 on acute stroke care in Belgium
}

\author{
Vincent Raymaekers ${ }^{1,2} \mathbb{D}$. Jelle Demeestere ${ }^{3} \cdot$ Flavio Bellante $^{4} \cdot$ Sofie De Blauwe ${ }^{5} \cdot$ Sylvie De Raedt ${ }^{6,7} \cdot$ Anne Dusart $^{4}$. \\ Lise Jodaitis ${ }^{9} \cdot$ Robin Lemmens $^{3} \cdot$ Caroline Loos $^{2,8}$. Ligot Noémie ${ }^{9} \cdot$ Matthieu P. Rutgers $^{10} \cdot$ Fenne Vandervorst $^{6,7}$. \\ Geert Vanhooren $^{5} \cdot$ Laetitia Yperzeele $^{2,8} \cdot$ Raul G. Nogueira $^{11} \cdot$ Thanh N. Nguyen $^{12} \cdot$ Peter Vanacker $^{1,2,8}$
}

Received: 26 April 2021 / Accepted: 10 June 2021 / Published online: 19 June 2021

(c) Belgian Neurological Society 2021

\begin{abstract}
A worldwide decline in stroke hospitalizations during the COVID-19 pandemic has been reported. Information on stroke care during the pandemic in Belgium is lacking. This study aims to analyze the impact of COVID-19 on acute stroke care in eight Belgian stroke centers. This Belgian study is part of an international observational and retrospective study in 70 countries and 457 stroke centers. We compared volumes of COVID-19 and stroke hospitalizations, intravenous thrombolysis and endovascular treatment rates, acute treatment time intervals and functional outcome at 90 days during the first wave of the pandemic to two control intervals (March-May 2019 and December-February 2020). From March 2020 to May 2020, 860 stroke patients were hospitalized. In the same time period, 2850 COVID-19 patients were admitted, of which 37 (1.3\%) were diagnosed with a stroke. Compared to the months prior to the pandemic and the same time epoch one year earlier, stroke hospitalizations were reduced (relative difference 15.9\% $[p=0.03]$ and $14.5 \%[p=0.05]$, respectively). Despite a reduction in absolute volumes, there was no difference in the monthly proportion of thrombolysis or endovascular treatment provided to the overall stroke hospitalizations. Acute treatment time metrics did not change between COVID-19 pandemic and control time epochs. We found no difference in 90-day functional outcomes nor in mortality after stroke between patients admitted during the pandemic versus control periods. We found a decline in the volume of stroke hospitalizations during the first wave of the COVID-19 pandemic in Belgium. Stroke care quality parameters remained unchanged.
\end{abstract}

Keywords COVID-19 pandemic $\cdot$ Stroke care $\cdot$ Belgium $\cdot$ Door-to-needle time $\cdot$ Door-to-groin puncture time

Vincent Raymaekers

vincent.raymaekers@azgroeninge.be

1 Department of Neurology, Groeninge Hospital, Kortrijk, Belgium

2 Faculty of Medicine and Health Sciences, Antwerp University, Antwerp, Belgium

3 Department of Neurology, Leuven University Hospital, Leuven, Belgium

4 Department of Neurology, Hôpital Civil Marie Curie, Charleroi, Belgium

5 Department of Neurology, Sint-Jan Hospital Bruges-Ostend, Bruges, Belgium

6 Department of Neurology, University Hospital Brussels, Jette, Belgium
7 Center of Neurosciences, University of Brussels, Brussels, Belgium

8 Department of Neurology, Antwerp University Hospital, Edegem, Belgium

9 Department of Neurology, Erasmus Hospital, Brussels, Belgium

10 Department of Neurology, Europe Hospitals, Brussels, Belgium

11 Department of Neurology, Grady Memorial Hospital, Emory University, Atlanta, GA, USA

12 Department of Neurology, Radiology, Boston Medical Center, Boston University School of Medicine, Boston, USA 


\section{Introduction}

In December 2019, a new severe acute respiratory syndrome coronavirus 2 (SARS-CoV-2) was isolated in Wuhan, China. The coronavirus disease 2019 (COVID19) potentially causes a severe acute respiratory syndrome with the need for long-term hospitalization including ventilation and intensive care. Healthcare systems all over the world were inevitably affected by the increasing demand in emergency departments and intensive care units [1]. Given the exponential increase in the number of infections and deaths, the World Health Organization (WHO) declared COVID-19 to be a pandemic as of March 2020 [2].

There have been numerous reports on neurological manifestations associated with SARS-CoV-2. These include encephalopathy, ischemic, haemorrhagic and cerebral venous stroke $[3,4]$. It is well known that infection associated inflammation leads to a prothrombotic state and subsequently to arterial or venous thromboses. Furthermore, it can be associated with other prothrombotic mechanisms including endothelial dysfunction, inflammatory cytokines and thrombotic diathesis $[5,6]$. The risk of severe disability and mortality appears to be higher in patients with COVID-19 associated strokes [7-9].

During the COVID-19 pandemic, a significant decline in acute stroke hospitalizations at a local, regional and national level was reported [10-13]. This is in contrast with the increasing reports of stroke in COVID-19 patients [14]. Recent international multicentric studies from Nogueira et al. concluded that there was a global decline in stroke hospitalizations, mechanical thrombectomy [15], intravenous thrombolysis (IVT) and interfacility IVT transfers with a greater magnitude of decline in centers with a higher COVID-19 hospitalization burden [16]. Information about the influence of healthcare-related and infrastructural changes of the COVID-19 pandemic on national acute stroke care in Belgium is lacking.

The aim of this study is to evaluate the effect of the COVID-19 pandemic in Belgium on acute stroke care metrics.

\section{Methods}

\section{Study design and population}

This study is a multicentric cross-sectional, observational, retrospective study. Data on stroke and COVID-19 admissions, IVT treatment and EVT treatment were collected from eight Belgian stroke centers. The stroke centers consisted of seven comprehensive stroke centers, capable of
24/7 neurosurgical and endovascular intervention. One center was a primary stroke unit with the ability to refer for endovascular intervention.

Median monthly door-to-needle time (DNT) and median door-to-groin puncture time (DGT) from the thrombectomy performing institute were collected for each center. We chose the first wave of the pandemic (March 2020 to May 2020) as the time epoch of interest. This epoch was compared with the 3 months directly preceding the first wave, the pre-pandemic period (December 2019 to February 2020), as the primary analysis and the corresponding time epoch in the prior-year period, (March 2019 to May 2019) as secondary outcome.

The study was reviewed and approved by each ethical committee in every stroke center and is conducted in accordance with the Declaration of Helsinki. There were no external funding sources.

\section{Study variables and outcomes measures}

Stroke hospitalizations consisted of either transient ischemic attack, ischemic stroke or intracerebral haemorrhage. IVT was defined as intravenous thrombolysis administration limited to acute ischemic stroke. Early treatment time window was defined as a time interval between symptom onset or last known well and IVT less than $4.5 \mathrm{~h}$. If the patient was last known well more than $4.5 \mathrm{~h}$ before treatment with IVT, it was referred to as late window or unknown onset. Data on the timing of the thrombolysis administration compared to symptom onset were collected as median per month for each center, as was the proportion of patients with thrombolysis within the first $4.5 \mathrm{~h}$ after the initial symptoms. Door-toneedle time (DNT) was defined as the time interval (in minutes) between arrival at the initial hospital and IVT bolus administration.

Patients treated with EVT with or without IVT were categorized as EVT treatment. Door-to-groin puncture time (DGT) was defined as the time interval (in minutes) between arrival at the EVT-capable hospital and arterial puncture. COVID-19 hospitalization was defined as of any patient admitted to the hospital with a positive nasopharyngeal polymerase chain reaction (PCR) test or radiological signs compatible with COVID-19.

Ninety days outcomes were measured using the modified Rankin Scale (mRS) to measure the degree of stroke related disability, ranging from no symptoms (0) to dead (6). The $\mathrm{mRS}$ for neurological disability has been proven to be a valid and reliable tool to assess the impact of strokes [17].

Overall stroke hospitalizations, proportion of IVT treatments, proportion early-window IVT, proportion of EVT treatments, median DNT and DGT were compared in the pandemic and pre-pandemic period. Next, the impact of the potential changes in stroke care during the pandemic 
on median mRS was compared in the same time intervals. Variables in the pandemic period were studied in comparison with the time interval in the previous year (March 2019 to May 2019).

\section{Statistics}

All statistical analyzes were performed with IBM SPSS version 27.0. Stroke hospitalizations were reported as mean \pm standard deviation (SD). Proportion of IVT and EVT compared to the total monthly stroke hospitalizations were presented as percentages. DNT and DGT were reported as median \pm interquartile range (IQR) by each stroke center independently. To analyze the difference in DNT and DGT the overall means of monthly median values were compared.

We used a Shapiro-Wilk test to assess normality of the data. Differences in monthly volumes between the different time periods for all continuous data were analyzed using a Mann-Whitney $U$ test. Proportional changes for the proportion of IVT and EVT within these time intervals were analyzed with a Chi-squared analysis. The analyzes were considered statistically significant if the $p$ value was $<0.05$.

\section{Results}

In Belgium, COVID-19 hospitalization peaked in April 2020 during the first wave of the pandemic. During the period from March 1 until May 30 2020, 2850 patients were hospitalized due to SARS-CoV-2 infection, across all participating centers.

\section{Stroke hospitalizations}

Figure 1 shows the evolution of COVID-19 and stroke hospitalizations in the three months before and during the first wave of the COVID-19 pandemic in Belgium. From March 1 until May 30, 2020, there were 860 stroke hospitalizations of which 37 strokes were registered in patients with COVID19. The total amount of stroke admissions in pre-pandemic period and prior-year period were 1023 and 1006, respectively. Compared with the pre-pandemic period, stroke hospitalizations were reduced by $19.9 \%$ during the first wave of the pandemic (95\% CI $-27.3 \%$ to $-4.4 \%, p=0.030)$. When compared to the prior-year period, stroke hospitalizations were reduced by $14.5 \%$ in the pandemic period $(95 \%$ CI $-24.4 \%$ to $-4.6 \%, p=0.045$ ) (Table 1 ).

During the first wave of the pandemic, mean monthly stroke hospitalizations (Tables 2, 3), decreased by $14 \%$ $(p=0.002)$ and $15.8 \%(p=0.025)$ compared to the prior year and the pre-COVID period, respectively.

\section{SARS-CoV-2 infections and stroke}

6 out of 8 participating centers provided data on SARSCoV-2 infections and hospitalizations during the first wave of the pandemic. In February, March, April and May 2020, respectively, 8, 1088, 1307 and 455 patients were hospitalized for COVID-19 in six hospitals. In total, $1.3 \%$ of COVID-19 hospitalizations (37 patients) were complicated by a stroke.

\section{Intravenous thrombolysis and door-to-needle time}

A total of 140, 223 and 191 patients received IVT during the prior year, the pre-COVID and the COVID periods, respectively. Total and monthly volume of patients treated with IVT did not change during the first wave of the pandemic (Tables 1, 2, 3). There was no difference in the monthly proportion of IVT in the prior year $(p=0.35)$ or pre-COVID period $(p=0.44)$ compared to the COVID-19 pandemic period. The proportion of patients treated with IVT within the first $4.5 \mathrm{~h}$ after symptom onset (early time window) remained stable during the pandemic period compared to the pre-COVID period and the prior year $([p=0.25]$ and $[p=0.25]$, respectively).
Fig. 1 Evolution of COVID-19 and stroke hospitalizations in the 3 months before and during the first COVID-19 wave in eight Belgian stroke centers. Left vertical axis represents COVID-19 hospitalizations. Right vertical axis indicates stroke hospitalizations. The amount of COVID-19 positive stroke hospitalizations is indicated for each month. "Asterisk" COVID-19+ stroke hospitalizations: February 2020: 1, March 2020: 10, April 2020: 15 and May 2020: 11
Stroke hospitalization

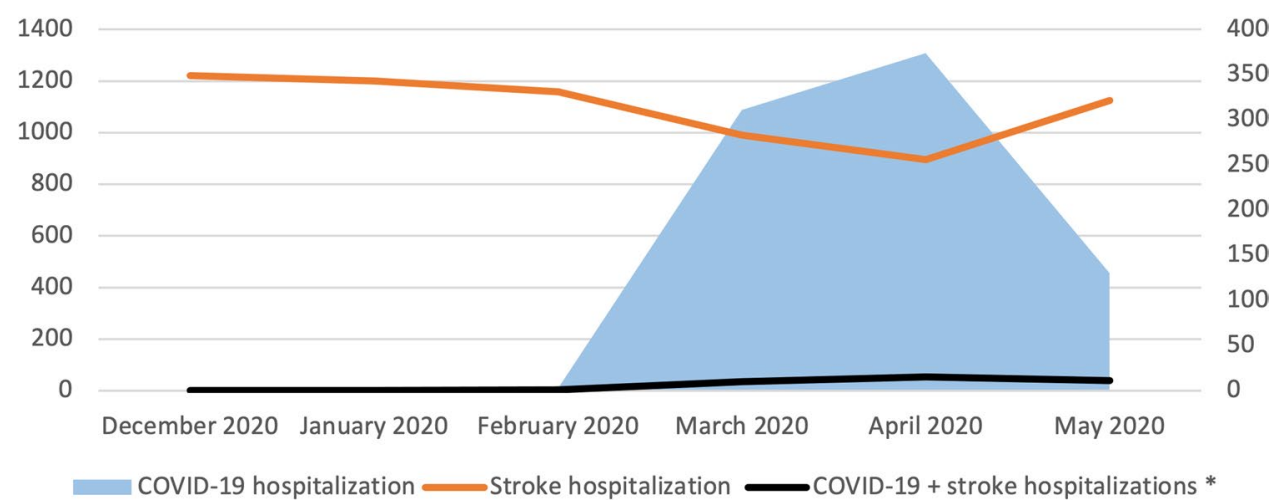


Table 1 Evolution of stroke hospitalizations and acute revascularization therapies during the first 3 months of the COVID-19 pandemic (A) compared with prior year: March 2019 until May 2019 and (B) compared with pre-COVID period: December 2019 until February 2020

\begin{tabular}{|c|c|c|c|c|c|c|c|c|}
\hline \multirow[t]{2}{*}{ Variables } & \multicolumn{4}{|c|}{ (A) Overall volumes prior year vs COVID period } & \multicolumn{4}{|c|}{ (B) Overall volumes pre-COVID vs COVID period } \\
\hline & Prior year & COVID period & Relative change & $p$ value & Pre-COVID & COVID period & $\begin{array}{l}\text { Relative change } \\
(95 \% \text { CI })\end{array}$ & $p$ value \\
\hline $\begin{array}{l}\text { Total stroke hospi- } \\
\text { talizations }(n)\end{array}$ & 1006 & 860 & $\begin{array}{l}-14.5 \%(-24.4 \% \\
-4.6 \%)\end{array}$ & 0.045 & 1023 & 860 & $\begin{array}{l}-15.9 \%(-27.3 \% \\
-4.4 \%)\end{array}$ & $\mathbf{0 . 0 3 0}$ \\
\hline Total IVT $(n)$ & 198 & 177 & $\begin{array}{l}-10.6 \%(-24.8 \% \\
3.6 \%)\end{array}$ & 0.200 & 207 & 177 & $\begin{array}{l}-14.5 \% \\
(-26.3 \% ;-2.7 \%)\end{array}$ & 0.055 \\
\hline Proportion IVT (\%) & $19.7 \%$ & $20.6 \%$ & $+0.9 \%$ & 0.310 & $20.2 \%$ & $20.6 \%$ & $+0.4 \%$ & 0.420 \\
\hline Total EVT (n) & 135 & 145 & $\begin{array}{l}+7.4 \%(-6.1 \% \\
10.9 \%)\end{array}$ & 0.280 & 166 & 145 & $\begin{array}{l}-12.7 \%(-35.6 \% \\
10.2 \%)\end{array}$ & 0.240 \\
\hline Proportion EVT (\%) & $13.4 \%$ & $16.9 \%$ & $+3.5 \%$ & 0.022 & $16.2 \%$ & $16.9 \%$ & $+0.7 \%$ & 0.350 \\
\hline
\end{tabular}

$I V T$ intravenous thrombolysis, $E V T$ endovascular treatment, $m R S$ modified Rankin' Scale (for neurological disability)

Table 2 Epidemiology and outcomes of acute revascularization therapies during the first wave of the COVID-19 pandemic compared to the similar period 12 months earlier (A) prior year: March 2019 until May 2019 and (B) COVID period: March 2020 until May 2020

\begin{tabular}{|c|c|c|c|c|}
\hline Variables & Monthly numbers & & & \\
\hline & Prior year ${ }^{(\mathrm{A})}$ & COVID period ${ }^{(B)}$ & $\begin{array}{l}\text { Relative } \\
\text { change } \\
(\%)\end{array}$ & $p$ value \\
\hline Stroke hospitalizations (mean, $\pm \mathrm{SD}$ ) & $41.9(1.9)$ & $35.8(4.0)$ & -14 & 0.002 \\
\hline IVT (mean, $\pm \mathrm{SD}$ ) & $8.7(5.1)$ & $8(3.9)$ & -8.6 & 0.250 \\
\hline Proportion IVT (\%) & $20.8 \%$ & $22.2 \%$ & +1.4 & 0.350 \\
\hline Proportion IVT early window (\%) & $86.4 \%$ & $88 \%$ & +1.6 & 0.250 \\
\hline $\operatorname{EVT}($ mean,$\pm \mathrm{SD})$ & $5.8(3.3)$ & $6.1(3.9)$ & +5.2 & 0.405 \\
\hline Proportion EVT (\%) & $13.7 \%$ & $17 \%$ & +3.3 & 0.220 \\
\hline \multicolumn{5}{|l|}{ Door-to-needle time } \\
\hline IVT early window (mean, $\pm \mathrm{SD} ;$ min) & $33.1(2.3)$ & $39.1(5.6)$ & +18.1 & 0.100 \\
\hline $\begin{array}{l}\text { IVT late window or unknown onset } \\
(\text { mean, } \pm \mathrm{SD} ; \mathrm{min})\end{array}$ & $45(0.7)$ & $129.1(89.1)$ & +186.9 & 0.002 \\
\hline Door-to-groin time (mean, $\pm \mathrm{SD}$; min) & $69.4(12.3)$ & $67.9(2.1)$ & -2.2 & 0.250 \\
\hline mRS 3 months (median) & 2 & 2 & $\mathrm{n} / \mathrm{a}$ & 0.440 \\
\hline
\end{tabular}

Early time window was defined as presentation in the hospital in the first $4.5 \mathrm{~h}$ after symptom onset $I V T$ intravenous thrombolysis, $E V T$ endovascular treatment, $m R S$ modified Rankin' Scale (for neurological disability)
The mean DTN in the early window was $33( \pm 2), 36$ $( \pm 5)$ and $39( \pm 6)$ minutes in the prior year (Table 2$)$, the pre-COVID (Table 3) and the COVID period, respectively. There was no significant change in the DNT between the different time epochs. In the late time window or in patients with unknown onset, the mean DNT was longer (129 vs $45 \mathrm{~min}$ ) in the COVID-19 period compared to the prior year $(+187 \%, p=0.002)$. Figure 2 illustrates the evolution of the DNT in the early window, the late window or unknown onset in relation to the volumes of acute revascularization therapy before and during the first COVID-19 wave. DNT in the late window or in patients with unknown onset was highly variable due to the small number of IVT in this time window (Fig. 2).

\section{Endovascular treatment and door-to-groin puncture time}

The total number of EVT was similar during the COVID19 period, compared to the prior year (146 vs 167 , $p=0.280$ ) and pre-COVID period (146 vs $167, p=0.240$ ). The overall proportion of patients treated with EVT across different centers was 3.5\% higher in the COVID-19 period compared to the prior year $(p=0.022)$. Mean monthly EVT per stroke center did not change over both time intervals (Tables 2, 3). Moreover, the monthly proportion of EVT in stroke patients did not differ between the two time intervals ( $p=0.220$ prior year and $p=0.460$ pre-COVID) and the COVID-19 period. 
Table 3 Epidemiology and outcomes of acute revascularization therapies during the first wave of the COVID-19 pandemic compared to the three months before (A) pre-COVID period: December 2019 until February 2019 and (B) COVID period: March 2020 until May 2020

\begin{tabular}{|c|c|c|c|c|}
\hline \multirow[t]{2}{*}{ Variables } & \multicolumn{4}{|c|}{ Monthly numbers } \\
\hline & Pre-COVID ${ }^{(A)}$ & COVID period ${ }^{(B)}$ & $\begin{array}{l}\text { Relative } \\
\text { change }(\%)\end{array}$ & $p$ value \\
\hline Stroke hospitalizations (mean, $\pm \mathrm{SD}$ ) & $42.5(1.1)$ & $35.8(4.0)$ & -15.8 & 0.025 \\
\hline IVT (mean, $\pm \mathrm{SD}$ ) & $9.3(4.2)$ & $8(3.9)$ & -14.0 & 0.130 \\
\hline Proportion IVT (\%) & $21.8 \%$ & $22.2 \%$ & +0.4 & 0.440 \\
\hline Proportion IVT early window (\%) & $86.2 \%$ & $88 \%$ & +1.8 & 0.250 \\
\hline $\mathrm{EVT}($ mean,$\pm \mathrm{SD})$ & $7(4.4)$ & $6.1(3.9)$ & -12.9 & 0.255 \\
\hline Proportion EVT (\%) & $16.3 \%$ & $17 \%$ & +0.7 & 0.460 \\
\hline \multicolumn{5}{|l|}{ Door-to-needle time } \\
\hline IVT early window (mean, $\pm \mathrm{SD} ;$ min) & $36.2(5.4)$ & $39.1(5.6)$ & +7.0 & 0.135 \\
\hline $\begin{array}{l}\text { IVT late window or unknown onset } \\
\quad(\text { mean }, \pm \mathrm{SD} ; \mathrm{min})\end{array}$ & $69.2(29.1)$ & $129.1(89.1)$ & +86.6 & 0.255 \\
\hline Door-to-groin time (mean, $\pm \mathrm{SD} ; \min )$ & $66.0(10.3)$ & $67.9(2.1)$ & +3.0 & 0.255 \\
\hline mRS 3 months (median) & 2 & 2 & $\mathrm{n} / \mathrm{a}$ & 0.455 \\
\hline
\end{tabular}

Early time window was defined as presentation in the hospital in the first $4.5 \mathrm{~h}$ after symptom onset $I V T$ intravenous thrombolysis, EVT endovascular treatment, $m R S$ modified Rankin' Scale (for neurological disability)

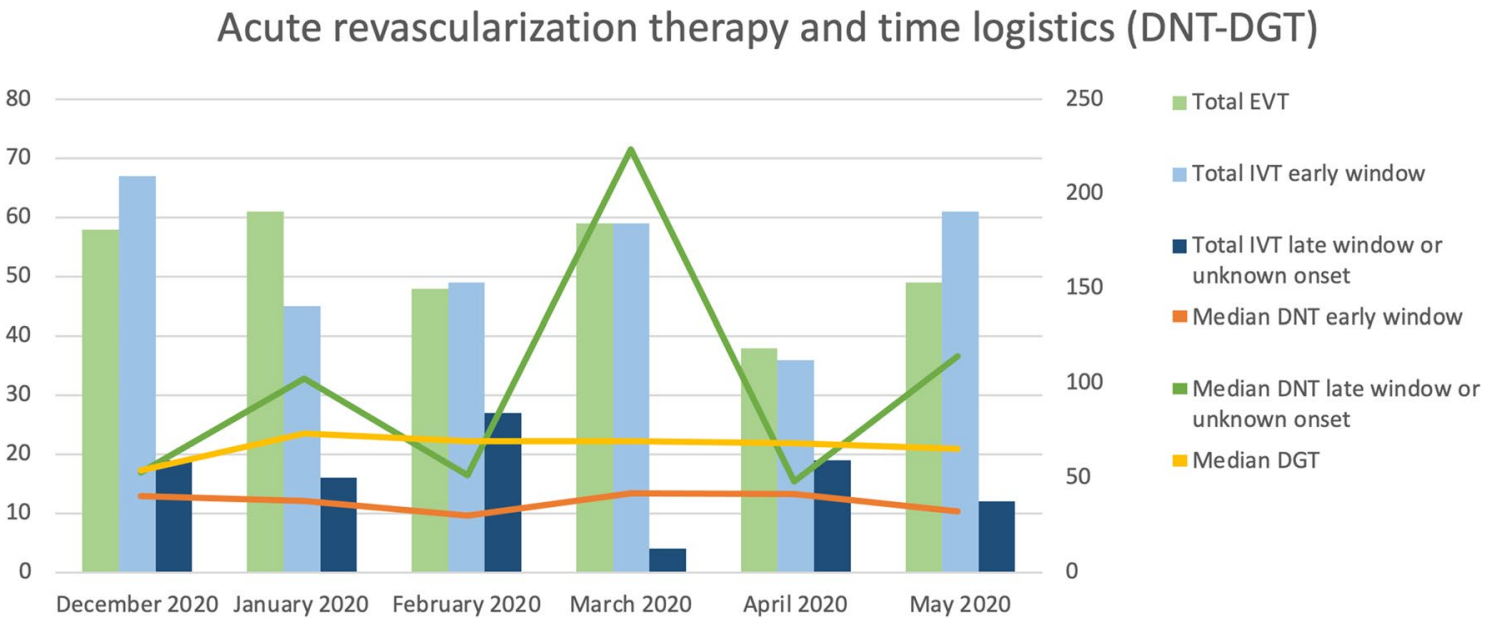

Fig. 2 Evolution of median door-to-needle time (DNT) in the early window, the late window or unknown onset and median door-to-groin puncture time (DGT) in relation to the volumes of acute revascularization therapy before and during the first COVID-19 wave in Bel-

Mean DGT was $68( \pm 2)$ minutes in the COVID-19 period, which was similar compared to $69( \pm 12)$ minutes $(p=0.25)$ in the prior year and $66( \pm 10)$ minutes $(p=0.26)$ in the pre-COVID period (Tables 2, 3). Figure 2 illustrates the evolution of the median DGT in relation to the volumes of acute revascularization therapy before and during the first COVID-19 wave in Belgium.

\section{0-days outcome}

We found no difference in stroke outcome after ninety days between patients treated during the first COVID-19 wave gium. Early time window for IV thrombolysis was defined as presentation in the hospital within $4.5 \mathrm{~h}$ after symptoms onset. The left vertical axis represents the COVID-19 hospitalizations. The right vertical axis indicates the median DNT and DGT values in minutes

(median mRS 2) and the prior year (median $\mathrm{mRS}=2$, $p=0.440$ ) or the pre-COVID period (median mRS 2 ; $p=0.455)$ (Tables 2 and 3).

\section{Discussion}

In this study on stroke care in 8 Belgian stroke centers during the first COVID-19 wave, we found a decline in stroke admissions of $15.9 \%$ compared to the three months prior to the pandemic and $14.5 \%$ compared to the same time interval the year prior to the pandemic. Acute revascularization 
treatment rates were unchanged. Furthermore, treatment time intervals also remained unchanged, although DNT for IVT was higher in the late treatment time window, possibly due to the small data sample. Outcome after 90 days was comparable for each time period with a median $\mathrm{mRS}$ of 2 .

This study was part of a large, international collaboration to collect worldwide observational data on acute stroke care during the COVID-19 pandemic. The study showed a worldwide decline of stroke hospitalizations (-11.5\%), EVT $(-12.7 \%)$ and IVT $(-13.2 \%)$ usage, regardless of COVID19 hospitalization burden $[15,16]$. This study shows that, despite a global decline in acute revascularization therapy, stroke care quality in Belgium was preserved during the first COVID-19 wave.

There have been numerous reports from different countries reporting similar results with a reduction of stroke hospitalisations ranging from 12 to $40 \%$, mostly compared with a time epoch prior to the first wave of the pandemic [18-27]. Other studies additionally found a decline in IVT or EVT treatments $[11,13,23]$. Rudilosso et al. describe a reduction in stroke admission and thrombectomies, particulary in the elderly population without affecting quality of care metrics [11]. Next to the reduction in mechanical thrombectomies, Kerleroux et al. notice an increase in imaging to groin puncture time in patients transfers to thrombectomies facilities, attributed to some extent of saturation of health transportation system [13]. Hsiao et al. reports a reduction of a $31 \%$ reduction in reperfusion treatment, primarily related to reduced thrombolysis rates. They hypothesized that the reduction is due to patients not seeking care at all or beyond $24 \mathrm{~h}$ after symptom onset more frequently [23].

During the first wave of the COVID-19 pandemic in Belgium, there was no change in total IVT treatments, nor in the proportion of patients treated with IVT. The total number of patients treated with EVT remained stable and the total EVT proportion increased $(+3.5 \%)$ during the COVID-19 period when compared to the prior year. Since only the number of stroke admissions decreased, one could hypothesize that fewer patients with transient and/or mild symptoms sought medical help or were referred by their general practitioner $[28,29]$. Next, the anxiety of getting infected with SARSCoV-2 in the hospital may have stopped patients to come to the hospital. Lockdown, lack of social contacts and physical distance requirements may have caused symptoms to go unrecognized. In contrast, better medication adherence and other lifestyle changes could have contributed to the decrease in stroke admissions. Similar trends were observed in cardiovascular medicine and aneurysmal subarachnoid hemorrhage admissions [30, 31]. Lastly, Nogueira et al. states that the decrease in global stroke admissions was also seen in centers with low or non-existent COVID-19 patients $[15,16]$.
We showed that the metrics on acute revascularisation therapy remained unchanged during the first wave of the COVID-19 pandemic in Belgium (Fig. 2). About 20-22\% of patients were treated with IVT, of which $86-88 \%$ were treated within $4.5 \mathrm{~h}$ after symptom onset. There was no change in door-to-needle or door-to-groin puncture time in the early time window. In contrast, Siegler et al. reports an increase in DNT associated with the COVID-19 pandemic, mainly attributed to a delay in the time between presentation and the ability to obtain cranial imaging [28]. Kler et al. state that an increase in EVT is a result of the increase in the time from first imaging until groin puncture due to operational issues [13]. We found no change in median mRS $(m R S=2)$ score after 90 days in the first wave of the pandemic. Changes in these treatment variables seem to be more variable than the decreased stroke hospitalization in different regions and countries. This could potentially be due to a difference in the extent of the pandemic in different regions or the diversity in COVID-19-related logistical changes in the hospitals.

In the 3 months of the pandemic in Belgium, 2850 COVID-19 patients were hospitalized in the participating centers. Stroke rate in COVID-19 patients was $1.3 \%$, which is in line with an overall incidence of $1.1-1.5 \%$ reported in international literature $[8,16]$. The COVID-19 pandemic is directly and indirectly accompanied with additional morbidity and mortality worldwide. Some recent reports indicate a significant increase in stroke morbidity, but not in mortality [32, 33]. Furthermore, COVID-19 patients with ischemic strokes seem to have more severe strokes and are more likely to have a worse functional outcome or result in death [34-36]. Patients with an ischemic stroke and comorbid COVID-19 infection have less favorable outcome and higher in-hospital death compared to COVID-19 negative patients [37]. More detailed information on COVID-19-associated strokes, such as stroke subtypes is currently lacking and a separate analysis on the outcome of acute stroke treatment in COVID-19 patients was not included, due to the small number of COVID-19 stroke patients.

\section{Study strengths and limitations}

This study is the first to describe the impact of the recent COVID-19 pandemic on stroke care in Belgium. It includes a multicentric pool of eight primary and comprehensive stroke centers in Belgium. The extent of variables included provides a comprehensive overview of changes in acute stroke care. The outcome measured with mRS after 90 days estimates the functional recovery before and during the first wave of the pandemic. However, data on the premorbid functional state or severity of stroke are lacking. Some variation in the proportions can be a result of different definitions (all strokes versus ischemic only) and populations included. 
The study has several limitations. The study sample is a variable combination of patients in main stroke centers and only one stroke unit, introducing a risk for selection bias. Acute stroke care in smaller hospitals could have been more affected by the local COVID-19 burden. The results of this study cannot be easily extrapolated to all Belgian acute stroke care. Additionally, there were no comparative analyses on COVID-19 patient volumes in the different stroke centers. The local burden of COVID-19 hospitalizations might have influences acute treatment metrics. Our study did not include patient data on demographics and stroke subtype (e.g. TIA, ischemic, haemorrhagic). In some stroke centers, the number of patients with a specific treatment (e.g. EVT) is low, increasing the risk of outliers in corresponding variables for the time logistics. Statistical analyses were not weighted for the COVID-19 hospitalization burden for each center individually.

\section{Conclusion}

The first wave of the COVID-19 pandemic was associated with a decline of $14-16 \%$ in the number of stroke hospitalizations in eight Belgian stroke centers. Acute revascularization therapy parameters for IVT or EVT remained unchanged. The overall quality of stroke care was preserved. These results provide guidance for future global or national healthcare-related setbacks.

Author contributions VR/PV/JD took the initiative, VR did perform statistics and wrote initial manuscript, all authors contributed to data collection, data interpretation and critical revision of the manuscript.

Funding None.

Availability of data and material Not applicable.

Code availability Not applicable.

\section{Declarations}

Conflict of interest None of the authors has a conflict of interest.

Ethics approval It is obtained in each contributing hospital.

Consent to participate Not applicable.

Consent for publication Not applicable.

\section{References}

1. Emanuel EJ, Persad G, Upshur R et al (2020) Fair allocation of scarce medical resources in the time of Covid-19. New Engl J
Med. 382(21):2049-2055. https://doi.org/10.1056/NEJMsb2005 114

2. WHO Director-General (2020) WHO Director-General's opening remarks at the media briefing on COVID-19 - 11 March 2020. https://www.who.int/director-general/speeches/detail/ who-director-general-s-opening-remarks-at-the-media-brief ing-on-covid-19---11-march-2020

3. Helms J, Kremer S, Merdji H et al (2020) Neurologic features in severe SARS-CoV-2 infection. New Engl J Med 382(23):2268 2270. https://doi.org/10.1056/NEJMc2008597

4. Abdalkader M, Shaikh SP, Siegler JE et al (2021) Cerebral venous sinus thrombosis in COVID-19 patients: a multicenter study and review of literature. J Stroke Cerebrovasc Dis 30(6):105733. https://doi.org/10.1016/j.jstrokecerebrovasdis. 2021.105733.PubMedPMID:33743411;PubMedCentralPMCID: PMCPMC7931726.eng

5. Hernández-Fernández F, Sandoval Valencia H, Barbella-Aponte RA et al (2020) Cerebrovascular disease in patients with COVID-19: neuroimaging, histological and clinical description. Brain 143(10):3089-3103. https://doi.org/10.1093/brain/awaa2 39 (PubMedPMID:32645151;PubMedCentralPMCID:PMC PMC7454411.eng)

6. Elkind Mitchell SV, Boehme Amelia K, Smith Craig J et al (2020) Infection as a stroke risk factor and determinant of outcome after stroke. Stroke 51(10):3156-3168. https://doi.org/10. 1161/STROKEAHA.120.030429

7. Tsivgoulis G, Katsanos Aristeidis H, Ornello R et al (2020) Ischemic stroke epidemiology during the COVID-19 pandemic. Stroke 51(7):1924-1926. https://doi.org/10.1161/STROK EAHA.120.030791

8. Siegler JE, Cardona P, Arenillas JF et al (2020) Cerebrovascular events and outcomes in hospitalized patients with COVID19: The SVIN COVID-19 Multinational Registry. Int J Stroke 30:1747493020959216. https://doi.org/10.1177/1747493020 959216 (PubMedPMID:32852257;PubMedCentralPMCID :PMCPMC7533468.eng)

9. Ntaios G, Michel P, Georgiopoulos G et al (2020) Characteristics and outcomes in patients with COVID-19 and acute ischemic stroke. Stroke 51(9):e254-e258. https://doi.org/10. 1161/STROKEAHA.120.031208

10. Zhao J, Li H, Kung D et al (2020) Impact of the COVID19 epidemic on stroke care and potential solutions. Stroke 51(7):1996-2001. https://doi.org/10.1161/STROKEAHA.120. 030225

11. Rudilosso S, Laredo C, Vera V et al (2020) Acute Stroke Care Is at Risk in the Era of COVID-19. Stroke 51(7):1991-1995. https:// doi.org/10.1161/STROKEAHA.120.030329

12. Kristoffersen ES, Jahr SH, Thommessen B et al (2020) Effect of COVID-19 pandemic on stroke admission rates in a Norwegian population. Acta Neurol Scand 2020142(6):632-636. https://doi. org/10.1111/ane.13307

13. Kerleroux B, Fabacher T, Bricout N et al (2020) Mechanical thrombectomy for acute ischemic stroke amid the COVID-19 outbreak. Stroke 51(7):2012-2017. https://doi.org/10.1161/STROK EAHA.120.030373

14. Ma A, Kase CS, Shoamanesh A et al (2021) Stroke and Thromboprophylaxis in the Era of COVID-19. J Stroke Cerebrovasc Dis 30(1):105392-105392. https://doi.org/10.1016/j.jstrokecerebrov asdis.2020.105392.PubMedPMID:33130478;eng

15. Nogueira RG, Abdalkader M, Qureshi MM et al (2021) Global impact of COVID-19 on stroke care. Int J Stroke 29:1747493021991652. https://doi.org/10.1177/1747493021 991652 (PubMedPMID:33459583;PubMedCentralPMCID: PMCPMC8010375.eng)

16. Nogueira RG, Qureshi MM, Abdalkader M et al (2021) Global Impact of COVID-19 on stroke care and intravenous thrombolysis. 
Neurology. https://doi.org/10.1212/wnl.0000000000011885 (PubMedPMID:33766997; eng)

17. Banks Jamie L, Marotta CA (2007) Outcomes validity and reliability of the modified rankin scale: implications for stroke clinical trials. Stroke 38(3):1091-1096. https://doi.org/10.1161/01. STR.0000258355.23810.c6

18. Yaghi S, Ishida K, Torres J et al (2020) SARS-CoV-2 and Stroke in a New York Healthcare System. Stroke 51(7):2002-2011. https://doi.org/10.1161/STROKEAHA.120.030335

19. Uchino K, Kolikonda Murali K, Brown D et al (2020) Decline in stroke presentations during COVID-19 surge. Stroke 51(8):25442547. https://doi.org/10.1161/STROKEAHA.120.030331

20. Siegler JE, Heslin ME, Thau L et al (2020) Falling stroke rates during COVID-19 pandemic at a comprehensive stroke center. J Stroke Cerebrovasc Dis 29(8):104953-104953. https://doi.org/10.1016/j.jstrokecerebrovasdis.2020.104953 (PubMedPMID:32689621;eng)

21. Sacco S, Ricci S, Ornello R et al (2020) Reduced Admissions for Cerebrovascular Events During COVID-19 Outbreak in Italy. Stroke 51(12):3746-3750. https://doi.org/10.1161/strokeaha. 120.031293.PubMedPMID:33059543;PubMedCentralPMCID: PMCPMC7678662.eng

22. Rinkel LA, Prick JCM, Slot RER et al (2021) Impact of the COVID-19 outbreak on acute stroke care. J Neurol 268(2):403408. https://doi.org/10.1007/s00415-020-10069-1

23. Hsiao J, Sayles E, Antzoulatos E et al (2020) Effect of COVID-19 on Emergent Stroke Care. Stroke 51(9):e2111-e2114. https://doi. org/10.1161/STROKEAHA.120.030499

24. Hoyer C, Ebert A, Huttner HB et al (2020) Acute Stroke in Times of the COVID-19 pandemic: a multicenter study. Stroke 51(7):2224-2227. https://doi.org/10.1161/strokeaha.120.03039 5PubMedPMID:32516064; eng (PubMedPMID:32516064;eng)

25. Gdovinová Z, Vitková M, Baráková A et al (2020) The impact of the COVID-19 outbreak on acute stroke care in Slovakia: Data from across the country. Eur J Neurol. https://doi.org/10.1111/ ene.14640 (PubMedPMID:33185918;eng)

26. Diegoli H, Magalhães PSC, Martins SCO et al (2020) Decrease in Hospital admissions for transient ischemic attack, mild, and moderate stroke during the COVID-19 era. Stroke 51(8):2315-2321. https://doi.org/10.1161/strokeaha.120.030481 (PubMedPMID:3 2530738;PubMedCentralPMCID:PMCPMC7302100.eng)

27. Desai SM, Guyette FX, Martin-Gill C et al (2020) Collateral damage - Impact of a pandemic on stroke emergency services. J Stroke Cerebrovasc Dis 29(8):104988-104988. https://doi.org/10.1016/j. jstrokecerebrovasdis.2020.104988.PubMedPMID:32689650;eng (PubMedPMID:32689650; eng)

28. Siegler JE, Zha AM, Czap AL et al (2021) Influence of the COVID-19 Pandemic on treatment times for acute ischemic stroke: the society of vascular and interventional neurology multicenter collaboration. Stroke 52(1):40-47

29. Wu Y, Chen F, Wang Z et al (2020) Reductions in Hospital admissions and delays in acute stroke care during the pandemic of COVID-19 [Original Research]. Front Neurol. https://doi.org/ 10.3389/fneur.2020.584734 ((English)@)

30. Mafham MM, Spata E, Goldacre R et al (2020) COVID-19 pandemic and admission rates for and management of acute coronary syndromes in England. Lancet 396(10248):381-389. https://doi. org/10.1016/S0140-6736(20)31356-8

31. Nguyen TN, Haussen DC, Qureshi MM et al (2021) Decline in subarachnoid haemorrhage volumes associated with the first wave of the COVID-19 pandemic. Stroke Vasc Neurol. https://doi.org/ 10.1136/svn-2020-000695 (PubMedPMID:33771936;PubMed CentralPMCID:PMCPMC8006491.eng)

32. Friedlich D, Newman T, Bricker S (2021) Impact of the COVID19 pandemic on stroke epidemiology and clinical stroke practice in the US. J Stroke Cerebrovasc Dis. https://doi.org/10.1016/j.jstro kecerebrovasdis.2021.105639 (PubMed PMID: 105639)

33. Grewal P, Pinna P, Hall JP et al (2020) Acute Ischemic Stroke and COVID-19: Experience From a Comprehensive Stroke Center in Midwest US. Front Neurol 11:910-910. https://doi. org/10.3389/fneur.2020.00910.PubMedPMID:32973666;eng (PubMedPMID:32973666;eng)

34. Fuentes B, Alonso de Leciñana M, García-Madrona S et al (2021) Stroke Acute management and outcomes during the COVID-19 outbreak. Stroke 52(2):552-562. https://doi.org/10.1161/STROK EAHA.120.031769

35. Calmettes J, Peres R, Goncalves B et al (2021) Clinical outcome of acute ischemic strokes in patients with COVID-19. Cerebrovasc Dis. https://doi.org/10.1159/000514562

36. Perry RJ, Smith CJ, Roffe C et al (2021) Characteristics and outcomes of COVID-19 associated stroke: a UK multicentre casecontrol study. J Neurol Neurosurg Psychiatry 92(3):242. https:// doi.org/10.1136/jnnp-2020-324927

37. de Havenon A, Yaghi S, Mistry EA et al (2020) Endovascular thrombectomy in acute ischemic stroke patients with COVID19: prevalence, demographics, and outcomes. J Neurointerv Surg 12(11):1045-1048. https://doi.org/10.1136/neurintsurg2020-016777.PubMedPMID:32989032;PubMedCentralPMCID: PMCPMC7523171.eng (PubMedPMID:32989032;PubMedCe ntralPMCID:PMCPMC7523171.eng)

Publisher's Note Springer Nature remains neutral with regard to jurisdictional claims in published maps and institutional affiliations. 